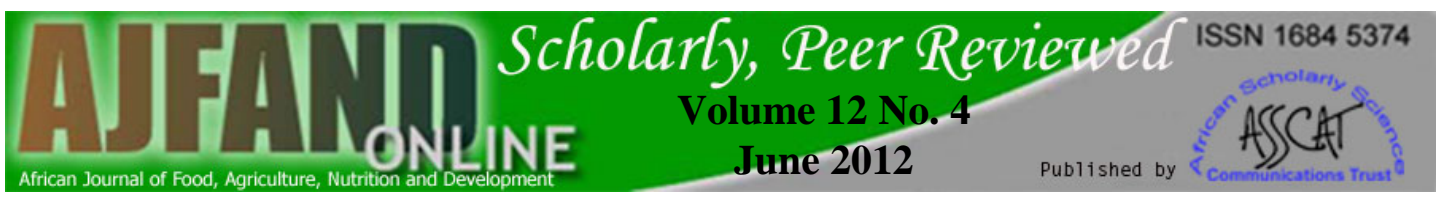

\title{
LANDSCAPE ANALYSIS - ASSESSING COUNTRIES' READINESS TO SCALE UP NUTRITION ACTIONS IN THE WHO AFRICAN REGION
}

\author{
Trübswasser $\mathbf{U}^{{ }^{*}}$, Nishida $\mathrm{C}^{2}$, Engesveen $\mathrm{K}^{3}$ and F Coulibaly-Zerbo ${ }^{4}$
}

*Corresponding author email: truebswasser@gmx.at

${ }^{1}$ Formerly with WHO/AFRO Food Safety and Nutrition Programme, Inter Country Support Team East and Southern Africa, Harare, Zimbabwe.

${ }^{2}$ World Health Organization, Nutrition, Health and Development Department, Geneva, Switzerland.

${ }^{3}$ World Health Organization, Nutrition, Health and Development Department, Geneva, Switzerland.

${ }^{4}$ WHO/AFRO, Food Safety and Nutrition Programme, Inter Country Support Team West Africa, Ouagadougou, Burkina Faso. 


\section{ABSTRACT}

The purpose of this paper is to describe the use of the Landscape Analysis to assess strengths and weaknesses in combating malnutrition in Sub-Saharan Africa. The Landscape Analysis is an inter-agency initiative to assess gaps and constraints and to identify opportunities for effective nutrition actions in order to accelerate intersectoral action for improving nutrition. In-depth Country Assessments to evaluate countries' "readiness" to scale up nutrition action have been conducted since 2008 in Sub-Saharan Africa. "Readiness" was assessed in terms of the commitment and capacity of each country and the focus was high stunting burden countries. The main focus was countries with heavy burden of undernutrition. From 2008 to September 2011, a total of 14 countries had undertaken the Landscape Analysis Country Assessment. Nine of them were in Africa: Burkina Faso, Comoros, Cote d'Ivoire, Ethiopia, Ghana, Madagascar, Mali, Mozambique, and South Africa. Three additional countries (Guinea, Namibia and Tanzania) were also planning to complete the Landscape Analysis Country Assessment in early 2012.

From the findings in the nine countries, the following recommendations have been made:

- Existing nutrition architectures and coordination mechanisms should be strengthened and better utilized;

- Nutrition needs to be mainstreamed and integrated in relevant sector policies;

- Advocacy at high levels is needed to highlight the importance of the lifecourse perspective, focusing particularly on nutrition interventions from preconception until the first two years of life;

- National nutrition policies need to be translated into programmatic actions;

- Human resource capacity for public health nutrition needs to be built with high quality in-service trainings in the short-term and long-term strategies to provide pre-service trainings;

- Community-based outreach should be strengthened by using existing channels;

- National nutrition surveillance systems need to be strengthened to ensure adequate use of data for monitoring, evaluation, and planning purposes.

As part of the implementation of the 2010 World Health Assembly resolution on Infant and Young Child Nutrition (WHA 63.23) WHO has proposed a process to help countries in developing scale-up plans, which incorporates the Landscape Analysis Country Assessment as a tool to help countries in undertaking the initial context mapping. The countries that have already undertaken an in-depth Country Assessment, such as the Landscape Analysis Country Assessment, are ready to move forward in implementing the next steps in developing scaling-up plans to accelerate action in nutrition. At the same time, WHO will support additional countries that have expressed interest in conducting the Country Assessment.

Key words: Nutrition, Landscape analysis, Capacity, Commitment, Scaling-up. 


\section{BACKGROUND}

All children have the right, and the same potential, to grow and develop. Undernutrition can be rapidly eliminated if adequate maternal and child nutrition security is ensured. However, about a third of children in developing countries, or 171 million children under 5 years of age, are too short for their age [1]. Progress in reducing underweight (too low weight for age), while positive in all sub-regions of Africa except southern Africa, is still insufficient to meet Millennium Development Goal 1 (MDG1), Target $2^{1}$. Increasing the rate of progress is a key goal, as not achieving the undernutrition target will negatively impact progress on achieving all other MDGs.

The Landscape Analysis was initiated at the end of 2007 as part of WHO's efforts to strengthen its contribution, in tandem with governments and other partners ${ }^{2}$, toward the achievement of the MDGs [2]. The Lancet Series on Maternal and Child Undernutrition published in 2008 provided a unique advocacy opportunity to accelerate evidence-based action in nutrition and to initiate a broader harmonization of various actors [3].

The Landscape Analysis aims to assess countries' readiness to accelerate action in nutrition, particularly in the 36 high-burden countries ${ }^{3}$, where $90 \%$ of the world's stunted children live. Nineteen of the 36 high-burden countries are in sub-Saharan Africa, of which eight ${ }^{4}$ have undertaken a Country Assessment of the Landscape Analysis (Burkina Faso, Côte d'Ivoire, Ethiopia, Ghana, Madagascar, Mali, Mozambique, and South Africa). In addition to Comoros, which also has fairly high stunting rates $(>40 \%)$.

In addition to the in-depth Country Assessment, the Landscape Analysis had two other global components: 1) desk review to develop country typologies of "readiness", and 2) development of the Nutrition Landscape Information System $(\mathrm{NLIS})^{5}$ which among others, provides country profiles with key nutrition indicators drawn from WHO Global Nutrition Databases as well as other existing databases within $\mathrm{WHO}$ and in partner agencies.

\footnotetext{
${ }^{1}$ Reducing underweight by $50 \%$ by 2015.

${ }^{2}$ The partner agencies included Food and Agriculture Organization of the United Nations (FAO), the Global Alliance for Improved Nutrition (GAIN), Helen Keller International (HKI), the UN Standing Committee on Nutrition (SCN), United Nations Children's Fund (UNICEF), and the World Food Programme (WFP).

${ }^{3}$ These 36 high-burden countries were identified by Black et al. [4] in the Lancet Nutrition Series: Afghanistan, Angola, Bangladesh, Burkina Faso, Burundi, Cambodia, Cameroon, Côte D'Ivoire, Democratic Republic of the Congo, Egypt, Ethiopia, Ghana, Guatemala, India, Indonesia, Iraq, Kenya, Madagascar, Malawi, Mali, Mozambique, Myanmar, Niger, Nigeria, Nepal, Pakistan, Peru, Philippines, South Africa, Sudan, United Republic of Tanzania, Uganda, Vietnam, Yemen, Turkey and Zambia. ${ }^{4}$ Comoros, although facing high rates of stunting above the level of public health concern, was not included in the 36 high-burden countries as its population is too small to contribute to $90 \%$ of the global burden.

${ }^{5}$ The NLIS can be accessed at http://www.who.int/nutrition/nlis/en/index.html
} 


\section{METHODS}

The Landscape Analysis Country Assessment focuses above all on obtaining in-depth information on commitment and capacity within countries through a participatory data collection, analysis and consensus building workshop. ${ }^{6}$ It provides a process for country teams to identify gaps, constraints and opportunities for integrating and scaling-up new and existing effective nutrition actions in order to create inter-sectoral action for improving nutrition. The Landscape Analysis Country Assessment Tool package is built around an analytical framework with indicators for commitment and capacity to accelerate actions to reduce maternal and child undernutrition at national and various sub-national levels. The package consists of tools for planning and for data collection, summary, analysis and presentation. It includes nine questionnaires and checklists for central, provincial and district level, health facilities, Antiretroviral (ARV) clinics and NGOs [5]. The Country Assessment Tool package has recently been extended to provide further practical tools for the participatory analysis, facilitating the data analysis. As part of the preparations for the Landscape Analysis Country Assessment, the country teams review the tools, select which ones they will use, and adapt them to the national situation.

The Landscape Analysis Country Assessment is based on the concept of readiness analysis. "Readiness analysis" is frequently-used in the private sector to assess where investing resources is likely to give the greatest return and for determining how best to invest in order to yield the maximum benefits. The Landscape Analysis, therefore, was the first attempt to systematically assess the readiness to act, but also the readiness to change in the nutrition-related area of work. It explores attitudes and perception of stakeholders to assess their commitment and capacity to scale up nutrition interventions [6].

To understand the nature of the nutrition problems being faced by the countries, the indicators considered for the Landscape Analysis were grouped between "nutritional problems", including: 1) nutritional outcomes and the immediate and underlying level factors affecting these outcomes and 2) "readiness to accelerate progress in undernutrition reduction". "Readiness", in turn, would be a function of "commitment" and "capacity". In addition, "meta-indicators", which have an impact on nutrition actions including governance, gender and human rights, were also included in the analytical framework.

The Country Assessments were conducted in five phases (see Box 1) by an intersectoral country team of national and regional/international partners during the course

\footnotetext{
${ }^{6}$ A snapshot of the readiness indicators is provided in the Nutrition Information Landscape System (NLIS) country profiles, available for all countries from www.who.int/nutrition/nlis, which dynamically draws data from the WHO Global Nutrition Databases as well as other databases within WHO and in partner agencies.
} 
of one to two weeks. ${ }^{7}$ Country teams were led by the health ministries and included representatives from other ministries like agriculture; national institutes for nutrition, health, food security, information; academia; UN agencies (FAO, UNICEF, WFP and WHO, SCN); international and national NGOs (Hellen Keller International, Micronutrient Initiative, Save the Children, Alive, GAIN and Thrive, Action against Hunger); and the World Bank. The country teams selected the regions and districts for the Country Assessment based on selected criteria, such as nutrition and/or food security indicators. However, in Ivory Coast and South Africa the assessment was done in all regions. The country teams conducted interviews at national, regional, district and health facility level using questionnaires from the Country Assessment Tool package that the country team had adapted to the situation in the country. Through a participatory analysis of one or two full day round-table sessions, the country team reviewed the findings gathered from the questionnaires and interviews in terms of strengths and weaknesses. Analytical framework developed to facilitate the data analysis process. South Africa, however, used a computerized process for the analysis. The results and proposed recommendations were reviewed and agreed in a stakeholder consensus meeting, which included different government sectors, UN agencies, bilateral agencies, donors, NGOs and private sector.

Phase 1: Preparations before the assessment week begins

- Brief overview analysis of the nutrition situation through review of key political and nutrition survey documents;

- Mapping of key stakeholders for nutrition in the country and of existing, on-going nutrition-related programmes, projects and activities, as well as an analysis of the nutrition situation;

- Identify members of the country team;

- Selection of regions and districts to include in the assessment;

- Arrange field visits and interviews with stakeholders at national, regional and district levels;

- Review Country Assessment Tools to be adapted to national situation;

- Prepare stakeholder meetings: consensus meeting on the last day.

Phase 2: Field-based, qualitative rapid assessment by task force

- Interviews, focus group discussions, checks, etc. at national level and during field visits to two to three field sites by sub-teams.

Phase 3: Analysis of findings and recommendations

- Meeting of country team to discuss key findings on strengths and weaknesses of willingness and capacity according to analytical framework;

- Propose draft recommendations for presentation at consensus meeting.

\footnotetext{
${ }^{7}$ In a one week's assessment, the assessment team makes final preparations on Monday, data collection and field visits on Tuesday and Wednesday, participatory analysis on Thursday, and a stakeholders' consensus meeting on Friday at which recommendations and next steps are agreed.
} 
Phase 4: Consensus-building workshop

- Presentation of key findings;

- Discussion of key recommendations for reinforcing strengths, correcting weaknesses and testing new strategies.

Phase 5: Preparation of final report and agreed recommendations

- Prepare and disseminate final report;

- Develop action plan to move forward the follow-up process.

\section{Box 1: The Five Phases of the Country Assessment}

\section{RESULTS}

The Landscape Analysis Country Assessments were initially undertaken in three subSaharan countries: Burkina Faso, Ghana, and Madagascar in 2008. These Country Assessments were followed by five other countries between 2009-2011: Comoros in April 2009, South Africa in the period of February-May 2009, Mozambique in January 2010, Ethiopia in March 2010, Côte d'Ivoire in April 2010 and Mali in July 2011. Three additional countries (Guinea, Namibia and Tanzania) were planning to complete their Landscape Analysis Country Assessments in early 2012 as part of their efforts to develop scale-up plans.

\section{Nutritional profile of countries}

Fig. 1 shows the nutrition burden in the nine countries that conducted the Landscape Analysis Country Assessment. While wasting remains a challenge, stunting presents a more pressing and irreversible problem and continues to pose a public health challenge, threatening national development in sub-Saharan African countries. Stunting indicates nutritional deprivation early in life (from conception up to two years of age); stunting after the age of two is irreversible and has long-term effects on children's health and development.

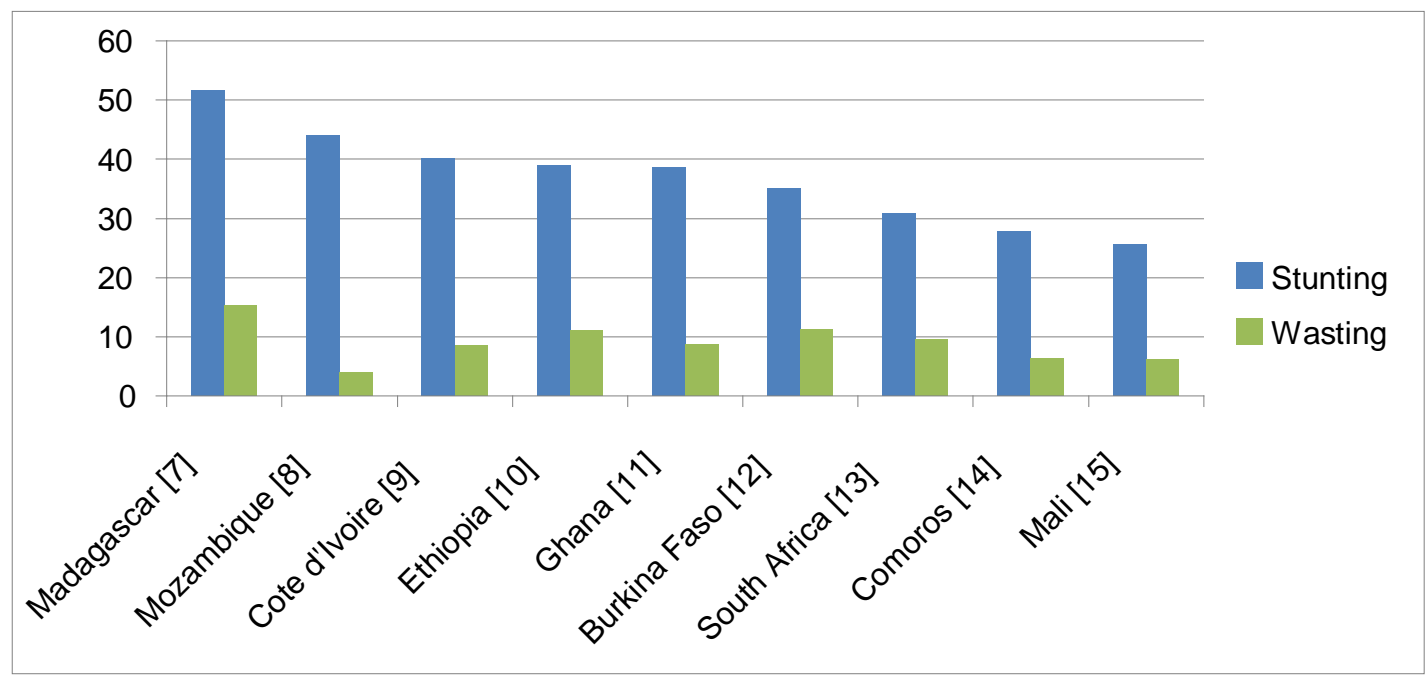

Figure 1: Stunting and wasting in children under 5 years in nine African countries 
While a few countries saw some improvements in nutrition indicators (such as Madagascar and Mali), stunting rates in those countries are still too high, for example, a stunting rate of $52 \%$ in Madagascar is still unacceptable [7, 15,16]. Other countries moved the other direction. In Côte d'Ivoire, however, stunting rates have increased from $32 \%$ in 2000 to $40 \%$ in 2006 [9]. Malnutrition also continues to be a major public health problem in Ethiopia and Mozambique with stunting rates of about $40 \%$ $[8,10]$.

South Africa may be considered an example of a country experiencing a nutrition transition, as there are concerns about both under- and over-nutrition in the country. The 2005 National Food Consumption survey (NFCS) for South Africa found that $18 \%$ of children between $1-9$ years were stunted, about $4,5 \%$ were wasted, and nearly $5 \%$ overweight [13].

\section{Findings related to commitment}

The Country Assessments found overall good commitment for nutrition in all countries. This can be seen by the position of nutrition departments within the government. In Ghana, for instance, the Nutrition Unit has been elevated to department level, implying more autonomy and resources for implementation. Another good example is Burkina Faso, where the Ministry of Health has turned the previous National Nutrition Center into the current Nutiriton Directorate, which is directly under the General Health Directorate.

Commitment to coordinate nutrition activities also seems to be in place in the assessed countries. Examples for coordination mechanisms on the national level include the National Directorate of Nutrition in South Africa and the Technical Secretariate for Food and Nutrition Security (SETSAN) in Mozambique.

However, the actual coordination between sectors and between government and partners seems to be a challenge in most countries. Findings from Burkina Faso, to discuss one such example, suggested that the anchorage of nutrition in the health sector might challenge the multisectorial approach. Coordination between national, regional and district level appeared to be a challenge in Ethiopia as well as Mozambique.

Policy documents and guidelines on nutrition are in place in all countries. The findings from South Africa indicated though that the multiplicty of guidelines and policies related to nutrition in the country may put pressure on provinces and districts resulting in the lack of focus and consequently hurting implementation. In Burkina Faso, Madagascar and Mozambique it was shown that nutrition-related policy documents and guidelines, as well as supplies (such as Vitamin supplements), necessary for implementation were often not available at district or health facility level, implying that policies are not operationalized or translated into programmatic actions. Another key finding is that while essential nutrition actions are implemented in some parts of the countries, the implementation rarely happens at a national scale. 
The commitment of governments was also assessed with regard to existence of separate nutrition budgets. Most countries did not even have a specific budget line for nutrition. Nevertheless, in some countries, like Burkina Faso, Ethiopia and South Africa, budgets for nutrition had increased both for government as well as development partners. However, priorities within governments sometimes shifted within a budget cycle and originally allocated nutrition funding was diverted to other programmes. Therefore, as it was found in the Madagascar assessment, programme budget for nutrition activities relies almost entirely on donors.

Another issue is that nutrition is not mainstreamed across sectors. While nutrition is included in national health strategies, such as the National Department of Health Strategic Plan of South Africa or the Child Health Policy of Ghana, nutrition is rarely tackled through policies from other, non-health sectors.

In most countries, the extent and severity of the nutrition problems were not clear to all stakeholders, and in particular, stunting is rarely recognized as a problem. For example, in the findings from South Africa, "nutrition programming” was mainly associated with provision of food supplements. In other countries, particularly in Madagascar and Mozambique, the Country Assessments found that there was little or no understanding that small stature, which affects most of the population, is often caused by chronic undernutrition, not genetics. This is likely due to the commonlyheld view across all the countries, that malnutrition can only be solved through increased food production, better education, and poverty reduction. Undernutrition was commonly seen as a humanitarian problem, not a developmental one, and was typically associated exclusively with the images of "starving" children. Furthermore, poor maternal nutrition was rarely seen as part of the problem of child malnutrition. Maternal nutrition programmes were weak or nonexistent in many countries, and there was a widespread failure to consider the lifecycle in the implementation of nutrition programmes. There was also a general lack of attention to the increasing double burden of malnutrition increasingly being experienced by the countries in Africa, and especially to the role of maternal and child undernutrition as risk factor for various non-communicable diseases later in life.

\section{Findings related to capacity}

In all countries, the Country Assessments showed that the human resource capacity for public health nutrition was inadequate with regard to distribution, appropriate skill set and motivation of staff. Particularly at the sub-national level, the shortage of staff was most evident. The assessment in Ghana found challenges to recruit and deploy graduate nutritionists at lower implementation levels. Technical officers trained in the Rural Health Training School in Nutrition and Disease Control seemed to put more priority on disease control rather than nutrition. Postgraduate nutrition degrees are available only to a limited extent in the counties assessed and most countries offer only dietetic training or brief trainings in nutrition as part of a bachelor degree programme. While training of existing health staff is done in all countries, most of the in-service trainings focus solely on management of acute malnutrition and rarely on more thorough nutrition-related training. 
Nutrition capacity on the community level was considered very weak. The findings from the Country Assessment of Burkina Faso for instance indicated that supervision and support for community workers are very poor and the lack of compensation affects sustainability, commitment and motivation.

Another crucial aspect that was observed in all countries was that nutrition information was often missing or poorly used. While nutrition information is being collected and used from Demographic Health Surveys, regular monitoring of nutrition indicators happens rarely. Most countries collect nutrition data through child growth monitoring or screenings for severe acute malnutrition. However, data management is often very poor; the quality of the collected information varies and data transfer is often delayed or incomplete, as was reported in the findings from Mozambique. Nutrition indicators are also lacking in national monitoring systems, like the National Health Information System in Burkina Faso, the Policy Planning, Monitoring and Evaluation Division in Ghana and the District Health Information Systems in South Africa. Unfortunately, these data are not always shared within the government or with partners. Therefore, data are rarely used for strategic planning purposes or for evaluation of programmes.

\section{First achievements in countries following the Country Assessment}

Following the assessment in Burkina Faso, all 13 regions have so far created Regional Nutrition Councils and have developed Regional Nutrition Action Plans.

Next steps after the Country Assessment in Comoros included the preparation of a first draft of a multi-sectoral nutrition policy and a first draft of a national Code of Marketing of Breast-milk Substitutes.

In Côte d'Ivoire, results and recommendations were presented to the members of the Landscape Analysis Steering Committee and development partners in a consensus meeting chaired by the Prime Minister's Advisor on Food Security and Nutrition.

The outcomes and recommendations have been guiding the development of a strategic action plan to reduce chronic malnutrition in Ethiopia.

As the next step after the Country Assessment, Ghana has been initiating the process of developing a Nutrition Policy to guide actions of all key players, taking into consideration of the outcomes of the Landscape Analysis Country Assessment.

Following the assessment in Madagascar, next nutrition strategic plan (PNAN II) as part of their efforts in developing scale-up plans is being developed.

In Mali, the findings of the Country Assessment served as one input in the process of developing a National Nutrition Policy and Action Plan.

Following the Country Assessment in Mozambique, a declaration of commitment was signed by the government, aiming at promoting efforts to reduce chronic malnutrition in the country and the development of a National Strategy for the Reduction of Chronic Malnutrition, which was launched in September 2010. 


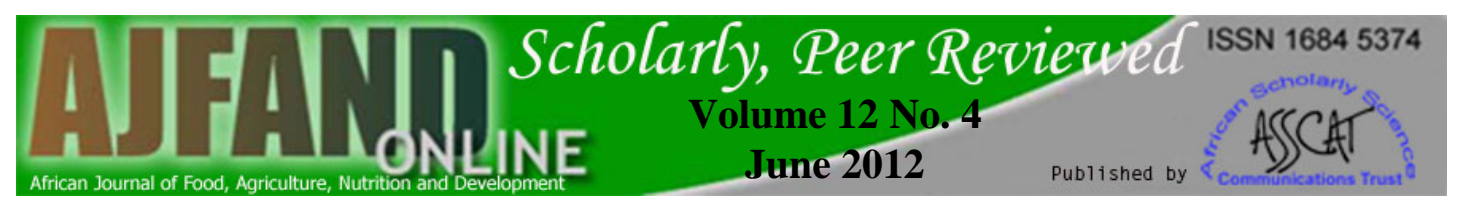

The findings of the Country Assessment in South Africa provided the department with the opportunity to develop a nutrition strategy.

\section{DISCUSSION AND CONCLUSION}

The Landscape Analysis Country Assessment provided a systematic approach to evaluate countries' readiness to accelerate action in nutrition, through the use of standardized assessment tools which were adapted to each country context. Findings of the Landscape Analysis Country Assessment may not be entirely new. However, a notable difference to previously used country assessment methodologies is the fact that the Country Assessment was conducted by an intersectoral/interagency county team in addition to the use of standardized, validated assessment tools. Furthermore, findings of the assessment were discussed, debated and agreed upon by all key stakeholders collectively in each of the countries at a stakeholder consensus meeting with the participation of high-level policy makers. This was a direct result of the participatory and inclusive process implemented through the Landscape Analysis.

The Landscape Analysis process also provided valuable opportunities, in particular for different levels within the government as well as with partners, to engage in rigorous discussions on the nutrition situation in countries and programmatic challenges. All countries stressed that participation of different sectors and stakeholders in the country was invaluable for ensuring commitment to take the recommendations from the Country Assessment forward.

Overall, the Country Assessments found a strong commitment to nutrition and nutrition policy frameworks, including nutrition coordination mechanisms and policy documents, existed in all countries. However, the coordination between nutrition partners is often weak and implementation of policies remains a challenge. Policies are often not operationalized and not translated into programmatic actions. Even if nutrition programmes are implemented, the implementation rarely happens at a national scale. The commitment of governments was also assessed with regard to existence of separate nutrition budgeting as most countries do not even have a specific budget line for nutrition. Despite the existence of budget lines for nutrition in a few countries, the programme budget for nutrition activities relies almost entirely on donors and not on governmental funds.

Another issue was that nutrition is not mainstreamed across sectors. This has been described previously, and is demonstrated by the weak commitment to nutrition in the Poverty Reduction Strategy Papers (PRSP) and United Nationas Development Assistance Framework (UNDAF) documents in the 36 high stunting burden countries referenced. This lack of commitment to nutrition in national development strategies can have serious consequences for human and economic development. This is because stunting is associated with lower educational achievement, cognitive ability, lower economic status and decreased productivity in adulthood [17].

In most countries, there was no common understanding of the extent and severity of the nutrition problems, and in particular, stunting was rarely considered a problem.

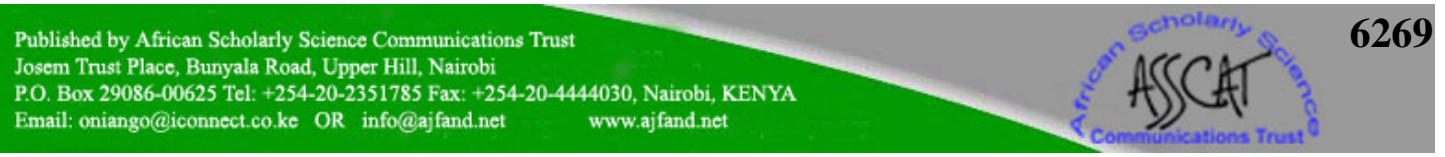


Furthermore, poor maternal nutrition was rarely seen as part of the reason for child malnutrition.

The human resource capacity for public health nutrition was insufficient in all the countries examined. Furthermore, community-based outreach of preventive nutrition action was often deemed not to be essential. This reflects the limited understanding of the chronic malnutrition burden in the countries assessed, which requires a long-term preventative approach as opposed to a reactive, curative one.

Another crucial aspect that was observed in all countries was that monitoring and evaluation was often missing or ineffective. Therefore, data are rarely used for strategic planning purposes or for evaluation of programmes.

Based on the findings from the nine assessed countries, the following recommendations can be made:

- Existing nutrition architectures and coordination mechanisms should be strengthened and better utilized before setting up a new separate mechanism;

- Nutrition needs to be mainstreamed and integrated in relevant sector policies to ensure contribution of all relevant sectors to improve nutrition countrywide;

- Advocacy at high levels is needed to highlight the importance of the lifecourse perspective, focusing particularly on nutrition interventions from preconception until the first two years of life;

- National nutrition policies need to be translated into programmatic actions, and those programmes must include concrete implementation and scale up plans;

- Human resource capacity for public health nutrition needs to be built with high quality in-service trainings of health workers in the short-term and long-term strategies to provide pre-service trainings of nutritionists and other health professionals;

- Community-based outreach should be strengthened by using existing channels, including from other sectors; awareness raising on chronic malnutrition and the need for preventative nutrition actions will be crucial;

- National nutrition surveillance systems need to be strengthened to ensure adequate use of data for monitoring, evaluation, and planning purposes.

Globally, there is currently a strong momentum to support the implementation of these recommendations on the country level. In May 2010, the $63^{\text {rd }}$ World Health Assembly adopted the resolution (WHA 63.23) on infant and young child nutrition [18]. It urged Member States, inter alia, to increase political commitment to preventing and reducing malnutrition in all its forms, to expedite implementation of the global strategy on infant and young child feeding, and to expand nutritional interventions. The World Health Assembly (WHA) also requested that the DirectorGeneral provide support to Member States in expanding their nutritional interventions 


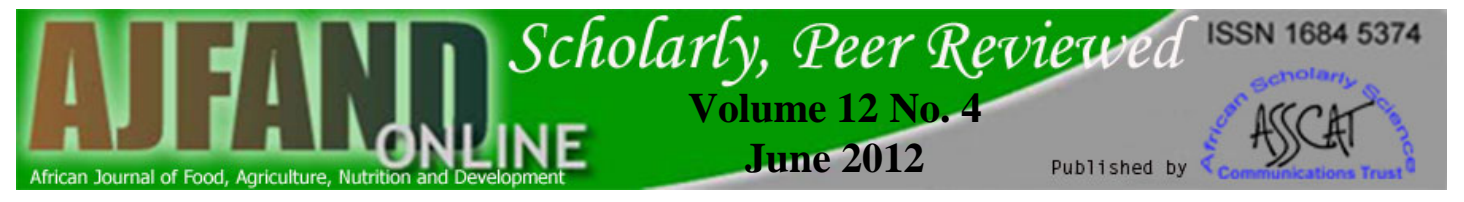

and to develop a comprehensive implementation plan on maternal, infant and young child nutrition as a critical component of a global multisectoral nutrition framework.

As part of the implementation of the WHA resolution and to support other on-going global and regional nutrition initiatives like REACH and $\mathrm{SUN}^{8}$, WHO has proposed a process which will guide countries "how" they may be able to develop country Scaling-up plans. This proposed process incorporates the Landscape Analysis Country Assessment as a first step in undertaking the initial scoping and context mapping for assessing existing implementation challenges in countries. Once the Country Assessment and context mapping are completed, countries would be ready to move forward in implementing the next steps in developing country scaling-up plans to accelerate action in nutrition.

A global movement on scaling-up nutrition action is increasing worldwide, but in particular in sub-Sahara Africa. There is need to seize the opportunity and the Landscape Analysis will be able to serve as a tool for countries to move forward in capitalizing this opportunity being presented.

\section{Acknowledgements}

The authors express their gratitude to all colleagues from the WHO Country Offices as well as the government and partner agencies involved in the Landscape Analysis Country Assessment in Burkina Faso, Comoros, Cote d'Ivoire, Ethiopia, Ghana, Madagascar, Mali, Mozambique and South Africa.

\footnotetext{
${ }^{8}$ REACH: Ending child hunger and undernutrition, http://www.reach-partnership.org; SUN: Scaling Up Nutrition http://www.unscn.org/en/nut-working/scaling up nutrition sun
} 


\section{REFERENCES}

1. World Health Organization. World Health Statistics, 2010.

2. Nishida C, Shrimpton $\mathbf{R}$ and I Darnton-Hill Landscape Analysis on countries'readiness to accelerate action in nutrition, SCN News \#37, 2009: 49.

3. Lancet Nutrition Series, Maternal and Child Undernutrition Study Group, 2008.

4. Black RE, Allen LH, Bhutta ZA, Caulfield LE, de Onis M, Ezzati M, Mathers $\mathbf{C}$ and $\mathbf{J}$ Rivera Maternal and child undernutrition: Global and regional exposures and health consequences, The Lancet 371, 2008: 243-60.

5. World Health Organization. Landscape Analysis Country Assessment Tools, Geneva, 2011.

6. Chopra M, Pelletier D, Witten C and M Dieterich Assessing countries' readiness: Methodology for in-depth country assessment, SCN News \#37, 2009: 17-22.

7. Institut National de la Statistique Ministere de l'Economie, des Finances et du Budget Antananarivo, Madagascar and ORC Macro Calverton, Maryland, USA, Demographic Health Survey, 2005.

8. National Statistics Institute Mozambique. Multiple Indicator Cluster Survey, 2009.

9. United Nations Children's Fund (UNICEF). National Institute of Statistics (Côte d'Ivoire). Côte d'Ivoire Multiple Indicator Cluster Survey 2006. New York, United States: United Nations Children's Fund (UNICEF).

10. Ethiopian Health and Nutrition Research Institute (EHNRI). National Nutrition Baseline Survey, Ethiopia, 2009.

11. Ministry of Health Republic of Ghana and Ghana Statistical Service and MEASURE DHS/Macro International Inc.., Multiple Indicator Cluster Survey, Ghana, 2006.

12. Ministry of Health, Nutrition Department. National nutrition survey, Ouagadougou Burkina Faso, 2009.

13. Labadarios D, Steyn NP, Maunder E, MacIntryre U, Gericke G, Swart R, Huskisson J, Dannhausera A, Vorster AA, Nesmvuni AE and JH Nel The National Food Consumption Survey (NFCS): South Africa, 1999, Public Health Nutrition: 8(5), 2005: 533-543. 
14. United Nations Children's Fund (UNICEF). Rapport d'évaluation de l'état nutritionnel des enfants de moins de 5 ans et de mortalité dans l'Union des Comores Décembre 2008.

15. Systeme d'alerte precoce (S.A.P). Enquete de base sur la sécurité alimentaire et la nutrition, Mali, 2007.

16. Cellule de Planification et de Statistique du Ministère de la Santé (CPS/MS), Direction Nationale de la Statistique et de l'Informatique (DNSI), ORC. Enquête démographique et de santé au Mali 2001. Demographic and Health Surveys. Calverton, Maryland, USA: CPS/MS, DNSI et ORC Macro, 2002 (and additional analysis).

17. Engesveen $\mathbf{K}$, Nishida $\mathbf{C}$, Prudhon $\mathbf{C}$ and $\mathbf{R}$ Shrimpton Assessing countries' commitment to accelerate nutrition action demonstrated in PRSPs, UNDAFs and through nutrition governance, SCN News \#37, 2009: 10-16.

18. World Health Organization. World Health Assembly Resolution WHA63.23 on infant and young child nutrition, Geneva, May 2010. 